\title{
¿Burbujas de filtro? Hacia una fenomenología algorítmica ${ }^{1}$
}

\section{Bubbles filter?
Towards an algorithmic phenomenology}

DOI: https://doi.org/10.18861/ic.2018.13.1.2836

\section{ANÍBAL ROSSI}

anibalrossi@gmail.com - Universidad Abierta Interamericana (UAI), Argentina.

Fecha de recepción: 12 de marzo de 2018

Fecha de aceptación: 25 de abril de 2018

\section{RESUMEN}

El presente trabajo reflexiona sobre las implicancias en la vida social de los sistemas algorítmicos que componen las diferentes plataformas digitales de acceso y participación enla web. El concepto deburbujas defiltrobrindado por Pariser (2011) es el punto de partida. El mismo es sometido a una lectura crítica con la intención de explicitar sus aristas más potentes, es decir, aquellas que abren camino a un amplio terreno de investigación sobre nuestra actual ecología mediática y en el que poco han incursionado los discursos académicos hispanoamericanos. Desde una mirada crítica se pone en crisis la metáfora de la burbuja y su capacidad heurística para aprehender, en sucomplejidad, elimpactodelos criteriosmaquínicos de selección en nuestras formas tecnológicas de vida (Lash, 2005). En su lugar, se propone el concepto de fenomenología algorítmica, concluyendo en la necesidad de una relación dialógica y/o metacomunicativa entre los usuarios y los sistemas algorítmicos en relación con los cuales los primeros experiencian el mundo. Finalmente, se exponen los resultados de un experimento empírico acotado en el que se indaga: por un lado, la veracidad de la hipótesis que presenta a Google como plataforma que aísla informativamente a sus usuarios y a DuckDuckGo como un buscador genérico; y, por otra parte, el grado de conocimiento y la disposición a intervenir las opciones de configuración ofrecidas por los buscadores.

PALABRAS CLAVE: burbujas de filtro, personalización de contenidos, buscadorgenérico, fenomenología algorítmica, metacomunicación.

\section{ABSTRACT}

Thispaperisareflexion of theimplications in the sociallife of algorithmic systems that make up the different digital platforms for access and participate take part of the web. The concept of filter bubbles provided by Eli Pariser in his TED conference in 2011 is the starting point. This is subjected toa critical reading with theintention of making explicititsmostpowerfuledges, that is, thosethatopen the way to a vast field of research on our current media ecology in which little has been ventured from the Hispanic American academic discourses.

After a condensed pointing out the key points that make up the posing of filter bubbles, it leads to a brief genealogy of "cookies". That is, the technological development that offered the essentialdigitalmnemictraces for theopening towards personalization of the contents on the web.

In the critical stage of work it is put in crisis the metaphor of the bubble and its heuristic capacity to apprehend in its complexity the impact of machinic selection criteria in our lifestyles that, today might say, they are technological lifestyles (Lash, 2004). Instead, he proposes the concept of algorithmic Phenomenology, concluding on the need for a dialogical and/or metacommunicative relationship between users and algorithmic systems on which they experiencetheworld. Finally presents the results of an empirical experiment of a limited nature in which it explores on the one hand, the truth of the hypothesis that presents Google as a platform that bubbles (isolates informatively) its users, and DuckDuckGoas ageneric search engine. As well as the degree of knowledge possessed by users of some configuration options offered by the search engines and how much they are willing to intervene them.

KEYWORDS: filterbubbles, contentcustomization, genericsearch engine, algorithmic phenomenology, metacommunication.

1 Unaprimera versión delartículo fuepresentada comoponencia en el VIIISeminario Regional(ConoSur)dela Asociación Latinoamericana de Investigadores de la Comunicación (ALAIC): “Políticas, actores y prácticas de la comunicación. Encrucijadas de la investigación en América Latina”, desarrollado los días 27 y 28 de agosto de 2015 en la Universidad Nacional de Córdoba (UNC), Argentina. 


\section{INTRODUCCIÓN}

El presente trabajo reflexiona sobre las implicancias que tienen en la vida social los sistemas algorítmicos que componen las diferentes plataformas digitales de acceso y participación en la web. Se toma como punto de partida el concepto de burbujas de filtro desplegado por Eli Pariser (2011;2017), sometiéndolo a una lectura crítica conlaintención de explicitar sus aristas más potentes, es decir, aquellas que abren camino a un amplio terreno de investigación sobre nuestra actual ecología mediática, donde poco han incursionado los discursos académicos hispanoamericanos. En tal sentido, en toda la primera parte del trabajo hacemos un señalamiento condensado de los puntos centrales que forman parte del planteo acerca de las burbujas de filtro, y realizamos una breve genealogía del desarrollo tecnológico quebrindólas huellasmnémicas digitalesindispensablesparala apertura hacia la personalización de los contenidos en la web: las cookies.

El artículo hace un análisis crítico de la metáfora de la burbuja y su capacidad heurística para aprehender en su complejidad el impacto de los criterios maquínicos de selección en las formas de vida actuales; que es posible afirmar, son formas tecnológicas de vida (Lash,2005). En su lugar, se propone el concepto de fenomenología algorítmica, concluyendo en la necesidad de una relación dialógica y/o metacomunicativa entre los usuarios y los sistemas algorítmicos en relación con los cuales los primeros experiencian el mundo.

Finalmente, en la última parte del trabajo, se exponen los resultados de un experimento empírico. Por un lado, se indaga la veracidad de la hipótesis que presenta a Google como plataforma que burbujea (aísla informativamente) a sus usuarios y a DuckDuckGo como un buscador genérico; por otro lado, se examina el grado de conocimiento que poseen los usuarios de algunas opciones de configuración ofrecidas por los buscadores y se observa en qué medida están dispuestos a intervenirlas. Este experimento fue realizado durante la primera mitad de 2015, en el marco del Seminario de Integración y Producción dela Licenciatura en Comunicación Social de la Universidad Nacional de Rosario (UNR), Argentina.

\section{BURBUJAS DE FILTRO}

En marzo de 2011 el estadounidense Pariser (2011)brindó una conferencia TED titulada "Cuidado con las burbujas de filtro en la web"; ese mismo año publicó el libro The filter bubble (Pariser, 2017). Su exposición, sobre todo, tuvo gran repercusión en Internet, incluso fue traducida a más de cuarenta idiomas y cuenta (hasta el momento de escribir este trabajo) con un número de visitas en la red superior a los cuatro millones. Quien hasta allí fuera reconocido, tan sólo, por su desempeño como activista político digital², llamó la atención de

2 Algunos de los proyectos de activismo político digital que cuentan con la participación de Pariser, cuando no es directamente uno sus fundadores, son MoveOn (véase: http://front.moveon.org/), Avaaz(véase: http://avaaz.org/ es/) y Upworthy (véase: https://www.upworthy.com/). 
muchos al poner en debate una cuestión poco referida y nulamente criticada desde un punto de vista socio técnico: las burbujas de filtro.

¿Qué son las burbujas de filtro? Según Pariser, el acceso a la información y a la participación en la web está condicionado por el diseño algorítmico de las plataformas utilizadas para ingresar a la misma. El concepto burbujas de filtro refiere al aislamiento informativo donde estarían quedando atrapados los usuarios como consecuencia de dichas configuraciones.

Desde 2009, año en que Google decidió tomarse más en serio el registro del comportamiento de sus usuarios para ofrecer resultados a medida ${ }^{3}$, Internet ha virado hacia la personalización de los contenidos. Desde entonces, los algoritmos no han dejado de conjeturar respecto de los usuarios: incesantemente se preguntan sobre los intereses de quien está detrás del navegador, a los fines de construir un perfil y, en función de esto, intentar predecir cuáles contenidos quiere ver y a cuáles publicidades podría ser más permeable. Para probar esta afirmación, basta simplemente realizar dos búsquedas en Google sobre un mismo tema desde distintos ordenadores ${ }^{4}$, yconstatarlas diferenciasentrelos resultados deamboscasos. Es decir, ante búsquedas idénticas obtenemos resultados variados.

Según el análisis de Pariser (2017) esto representa un grave problema. El peligro fundamental detrás de dicha divergencia, aparentemente sin importancia, es la atomización informativa. Las configuraciones maquínicas que promueven estas plataformas amenazan con operar una desconexión simbólica con los otros. Lapredicción delosintereses delosusuarios a partir delatraza desus movimientos pasados y basada tan sólo en el criterio de relevancia, atentaría contra la posibilidad de seguir habitando un universo simbólico compartido. Cercado en una suerte de condescendencia informativa a consecuencia de la reproducción de su recorrido previo, cada quién se encontraría preso en su propia burbuja.

\subsection{Burbujas y democracia}

La preocupación de Pariser sobre las burbujas de filtro involucra un amplio abanico de problemas como el control, la privacidad, la venta de datos, el refuerzo del sesgo de confirmación y la pérdida de iniciativa en el proceso de búsqueda. Sin embargo, su planteo acerca de los criterios de selección y organización de la información que utilizan Google y Facebook (entre otros), está fundamentalmente ligado a la cuestión de si estas configuraciones, tal como están dispuestas, son convenientes o no para un contexto democrático ${ }^{5}$. La

3 Según un artículo de Pickhardt (2011) son cincuenta y siete las señales que Google toma en cuenta para elaborar sus resultados de búsquedas.

4 El ejemplo utilizado por el autor para ilustrar este punto surge a partir del término "Egipto". Una excelente explicación sobre el problema de las burbujas de filtro a partir de este ejemplo se encuentra en: http://dontbubble.us/

5 La definición de democracia que retomamos aquí, de la mano del autor, implica la existencia de un espacio informativo común, la coexistencia de lo diverso y la posibilidad de participación, discusión e intervención de los criterios de construcción de ese espacio: "Los asuntos importantes que de forma indirecta afectan nuestra vida pero existen fuera de la esfera de nuestro propio interés inmediato son la piedra angular y la razón de ser de la democracia" (Pariser, 2017, p. 81). 
regla con la que juzga el papel que estas plataformas tendrían que cumplir en dicho contexto presupone una relación de continuidad con los medios masivos tradicionales. Así como lo hicieran estos medios pre-digitales, los actuales algoritmos deberían contribuir a la percepción de un espacio compartido y de una agenda informativa común, aunque "la democracia que trae Internet aún no ha llegado" (Pariser, 2011). Dentro de las burbujas de filtro los usuarios ven lo que los diferentes sistemas concluyen que quieren ver, y no lo que tienen que ver. Según Pariser, los usuarios no sólo no deciden lo que se les muestra sino, lo que juzga aún más grave, no tienen idea de lo que queda afuera.

Su propuesta, pretendidamente superadora de esa realidad descrita, aspira a la construcción de un debate en torno a la ética del código. Así como los gatekeepers de los medios masivos fueron en su momento sometidos a cuestionamientos similares, es necesario hoy apuntar a la inteligencia maquínica que constituye las plataformas de acceso a la información y a la participación en la web. Estima prioritario avanzar en dirección de un enriquecimiento cualitativo delas variables a partir delas cuales se ecualizan los criterios de personalización del contenido. No sólo la idea de relevancia sino, también, las de inconformidad, contenido estimulante e importante deberían ser parte de la ecuación. De igual manera, el análisis de lo que muestran y lo que dejan fuera los sistemas algorítmicos, los criterios a partir de los cuales trabajan, la información que disponen de los usuarios y el modo en que operan, resultan cuestiones que no pueden quedar ajenas a las discusiones sobre la ecología mediática actual.

Antes de concluir esta presentación del problema planteado por Pariser, resulta insoslayable el trazado de una genealogía que nos permita ubicar la emergencia de la tecnología (cookie) que habilitó el tipo de conjeturas maquínicas señalas arriba. Una genealogía mediática. La genealogía de una galleta, como le gustó decir al inventor de las cookies.

\subsection{Genealogía de una galleta}

Supo cavilar Borges (1994) que la verdadera historia suele ser pudorosa. Es decir, los hechos relevantes pueden pasar desapercibidos y sus fechas esenciales permanecer ocultas durante mucho tiempo. Y la historia de los medios en general, y el derrotero de Internet en particular, no son la excepción (Berners Lee \& Fischetti, 2000). Lou Montulli, tal como se describe en este apartado, fue protagonista de uno de esos hechos ocultos o poco visibles que han tardado en conocerse.

Corría el año 1994, tallo señala Schwartz (2001), cuando un equipo de desarrolladores de Netscape Communications, abocado a resolver la implementación de un carro de compra online para una aplicación de comercio electrónico, tuvo que afrontar un desafío técnico. Los representantes del cliente solicitaban encontrar una forma de guardar los estados de transacción en el ordenador de cada usuario, y no en el servidor de Netscape como se hacía habitualmente. 
Montulli, un programador de tan solo 23 años que formaba parte del equipo de desarrollo dela empresa, avistó una idea que podría resolver el inconvenientey diseñó una solución para lograr quelos ordenadores recordaran la información de cada sesión a la que denominó cookie. No se trataba de software ni de código, sino de datos almacenados en el ordenador del usuario a pedido del servidor web. A partir de este pequeño gran invento de Montulli, los navegadores y los sitios webs comenzaron a recordar a los internautas: así las cookies ofrecieron las huellas mnémicas con las que la web comenzó a construir a sus usuarios modelo. Esas escurridizas entidades situadas en la conjunción entre cuenta de usuario, ordenador y browser eran ahora conjeturadas. Que el navegador sepa el nombre de usuario antes de escribirlo, que no sea necesario loguearse cada vez que se ingresa a un sitio o que el buscador hable el idioma de cada usuario son tan solo algunas de las posibilidades habilitadas por este desarrollo.

Las Cookies se pueden diferenciar según:

El tiempo que permanecen activas:

- Persistentes: es decir, que tienen una fecha de caducidad definida a fin de sobrevivir a varias sesiones de navegación. Una vez cumplida esa fecha los datos registrados desaparecen.

- No persistentes: se eliminan cuando el navegador se cierra.

El seguimiento que hacen del usuario:

- Locales: sólo registran las acciones de los usuarios dentro del sitio.

- De terceros: registran los movimientos del usuario más allá de un sitio específico. Se usan generalmente con fines publicitarios ${ }^{6}$.

En ausencia de las cookies, cada petición de una página web o de alguno de sus componentes sería un evento aislado, sin relación con el resto delas peticiones de otras páginas del mismo sitio (Cobo, Gómez, Pérez \& Rocha, 2005).Ergo, esta tecnología representó, sin dudas, una notable innovación, aunque en el terreno de los medios, como lo supo ver McLuhan (1990), las innovaciones, los cambios, nunca son avances sino que se trata de desplazamientoslaterales sobre los múltiples puntos cardinales del espacio mediático, cuyos efectos responden a una dinámica compleja e indeterminada. Así fue que Montulli no imaginó (¿cómo podría?) que una simple galleta, como le gustaballamar a las cookies, se convertiría en el primer eslabón de una enrevesada cadena alimenticia digital donde la publicidad online, la venta de datos personales y las burbujas de filtro, se contarían entre las especies deformes de ese ecosistema.

6 Vale aclarar que las cookies también se distinguen de acuerdo al tipo de dato que registran: preferencias (idioma número de resultados visibles en una búsqueda, localización, etc.); seguridad (id, últimas conexiones, etc.): procesos (ligado al funcionamiento de los sitios web.); las relacionadas principalmente con la publicidad (Cobo, Gómez, Pérez, \& Rocha, 2005). 


\section{LOS ALGORITMOS SE HACEN VISIBLES}

Estudios pioneros en el área del análisis de redes como los de Barabási (2003) o Watts (2006), entre otros, hace tiempo que desfondaron la posibilidad de reproducción de ciertos discursos utópicos queveían en la web el reino delaigualdad.El 80/20 (o Ley de Pareto ${ }^{7}$ ) gobernante del reparto de links explicitaba que el carácter de su topología, como la de todas las redes libres de escala, se encontraba lejos de una distribución equitativa. No menos tiempo ha transcurrido desde que se ha vuelto manifiesta la existencia de regiones inexploradas por los buscadores. Zonas silvestres que forman parte de la anatomía oculta de la red: web profunda (en inglés, deep web) es el nombre con el que se la designa. No obstante, poco se había dicho hasta aquí sobre el comportamiento de Internet desde su trama algorítmica $\mathrm{O}$, al menos, no existía una discusión que ligue este tema con preocupaciones menos exclusivas de un tecnicismo ingenieril y más próximas a las ciencias sociales.

Gran parte de la puesta en cuestión a gran escala de los criterios maquínicos de curaduría informativa y sus posibles efectos se la debemos al trabajo de Pariser. A partir de allí, ha emergido un terreno de reflexión que es necesario explorar, cultivar y enriquecer.

\subsection{La metáfora de la burbuja y la burbuja de la metáfora}

Las metáforas son indispensables. En ocasiones abren horizontes de percepción y reflexión, posibilitando la creación de conceptos que permiten aprehender el mundo y habitarlo de múltiples maneras; pero nunca son inocentes. El aleph conceptual no existe: un concepto es lo que muestra, pero eso no es todo. Del mismo modo las metáforas, al tiempo que son productivas y hacen visibles diferentes situaciones, poco (o másbien nada) dicen deaquello conloqueson incompatibles.

La metáfora de la burbuja usada por Pariser pone el acento en el encierro y la atomización. No es ningún halago para los usuarios afirmar que viven en burbujas de filtro; por el contrario, el término invoca todas las objeciones del solipsismo. Como si el resultado de la personalización fuera la inmersión en una esfera informativa egocéntrica en la que tan solo circulan fragmentos estáticos de una identidad algorítmicamente conjeturada. La pérdida de un universo informativo común y la desconexión con los otros son los peligros sobre los cuales este término nos advierte. Como ya referimos, a modo de ejemplo sencillo, dos personas buscando lo mismo o visitando su página de inicio obtendrán resultados diferentes. Ya no existe un Google estándar; en Facebook no hay portada (Igarza, 2008).

En tal sentido, haciendo hincapié en la metáfora dela burbuja, vemos quela

7 La Ley de Pareto hace referencia a un patrón de distribución subyacente a un amplio horizonte de fenómenos entre los que se cuentan la repartición de la riqueza, las citas bibliográficas y la distribución de los enlaces en la web (entre otros). Lleva ese nombre en alusión al ingeniero parisino del siglo XIX Wilfredo Pareto. En un sentido muy esquemático, podría afirmarse que rige La ley Pareto siempre que un $80 \%$ de lo que se distribuye en una población queda en poder de un $20 \%$, mientras el $80 \%$ población se reparte el $20 \%$ restante. Para más detalles véase: Watts (2006). 
divergencia planteada es entendida en clave negativa, lo que se traduce en una especie de moral informativa: "vamos hacia una Internet que nos va a mostrar lo que cree que queremos ver y no lo que tenemos que ver" (Pariser, 2011).

\section{2. ¿Un buscador estándar?}

Haciéndose eco de las afirmaciones anteriores, han emergido varias alternativas a los buscadores más usados que prometen no rastrear los datos del usuario, ni utilizarlos con fines comerciales. Entre ellas se destaca DuckDuckGo, buscador de código abierto apoyado en una comunidad de contribuyentes que participan activamente para mejorarlo. Entrelas descripciones dela iniciativa se afirma como una de sus bondades que quienes utilicen DuckDuckGo tendrán en manos un buscador estándar: es decir, siguiendo nuestro ejemplo, dos usuarios diferentes haciendo la misma búsqueda, obtendrán los mismos resultados. La suposición de fondo es que los algoritmos deben imitar la premisa homogeneizante de los medios predigitales ofreciendo el mismo contenido para todos (broadcast). Lo que se intenta recuperar por esta vía es la ficción, instituida a partir de los medios masivos, de un contexto informativo compartido. Aquella idea de que los medios son como ventanas abiertas a un mundo que, aunque sujeto a interpretaciones, se pretende objetivo (García Moggia, 2014).

En relación a este punto, la operación de análisis muestra un carácter, si no fallido, al menos abierto a cuestionamientos. Intentar reconstituir antiguas premisas en vistas de su crisis de validez puede obturar la visión de mundos posibles, rupturistas respecto del pasado, pero cargados de positividad. Quizás el tipo subjetivo que estos pretenden instituir no sea compatible ya con la idea estatal de un único centro configurante o, en este caso, un único centro informativo (Lewkowicz, 2004). De esta manera, prestar atención al presente, leyéndolo con atención y reconociendola dureza de épocas anteriores, es la clavefundamental para no examinarlo exclusivamente bajo el signo dela pérdida ${ }^{8}$.

Es innegable la importancia que comporta, en el ámbito de las ciencias sociales, el establecimiento de discusiones en torno a los algoritmos de filtro y el modo en que conjeturan a los usuarios para tejer sus horizontes informativos. Aunque la promesa de retorno siempre es tentadora, resulta necesaria la construcción de nuevas figuras conceptuales que habiliten reflexionar sobrela ecología mediática actual, atendiendo a sus rasgos singulares. Quizás la democracia de Internet, desgarrada porla tensión entrelas figuras del consumidoryla del ciudadano, no haya llegado aún. Oacaso nuncalleguesisela espera como repetición deloya conocido (Lewkowicz, 2004). Tal es el riesgo detrás del concepto de burbuja de filtro.

8 Entre las muchas alternativas que se podrían mencionar y que sitúan la personalización de contenidos en un terreno diferente del fenómeno de las burbujas de filtro, podemos destacar: Nuzzel (http://nuzzel.com/). Esta plataforma ofrece una curaduría informativa redológica en múltiples capas constituida por aquello que comparten los contactos de un usuario determinado y lo que comparten los contactos de los contactos de ese usuario. Incluso es permeable a la posibilidad de entrar en las constelaciones de cada usuario y ver qué comparten sus contactos y los contactos de sus contactos. 


\section{HACIA UNA FENOMENOLOGÍA ALGORÍTMICA}

Las preguntas sobre lo que las plataformas digitales muestran, lo que dejan fuera y a partir de qué criterios, se inscriben en un registro diferente al del análisis de los medios atravesado por el par mentira/verdad y la centralidad del contenido. Cuando Pariser (2011) afirma que no son los usuarios quienes deciden qué entra en la pantalla de inicio o la página de resultados de una búsqueda, está dejando en claro que el problema no está directamente ligado a qué, a cómo ni a quién de lo que se dice. La veracidad o confiabilidad de los contenidos no corre a la par de este cuestionamiento: en dicho análisis no opera el supuesto hermenéutico de que hay un significado oculto que no se hace evidente y requiere, por tanto, ser descifrado. La sospecha se dirige más bien hacia otro horizonte: indagar cuáles son las condiciones de posibilidad que hacen que un entorno informativo dado aparezca. Así se sitúa el problema del acceso a la información en un terreno fenomenológico.

Para esclarecer este punto resulta de gran auxilio remitir a una definición del concepto de fenomenología, desarrollado por Deleuze(2008), según la cual "hay fenomenología a partir del momento en que el fenómeno ya no es definido como apariencia sino como aparición (...). La aparición es lo que aparece en tanto que aparece" (p. 26). A lo que Deleuze agrega:

\footnotetext{
La fenomenología se plantea la pregunta: ¿qué es el hecho de aparecer? Es lo contrario de una disciplina de las apariencias. La apariencia es algo que remite a la esencia dentro de una relación de disyunción, dentro de una relación disyuntiva: o bien pertenece a la apariencia, o bien pertenece a la esencia. ¿A qué remite la aparición? La aparición es muy diferente, es algo que remite a las condiciones de lo que aparece (Ibídem).
}

Claramente el planteo del problema de las burbujas de filtro se corresponde con la relación conjuntiva entre lo que aparece ylas condiciones de posibilidad de lo que aparece. El hecho de que esa condición de posibilidad esté atravesada por una configuración tecnológica constituye un territorio fenomenológico singular. No se trata ya de una fenomenología a secas, sino de una fenomenología tecnológica (Lash, 2005). Algorítmica, para ser más precisos.

En esta fenomenología, lo que aparece, lo que se experiencia, está atravesado por la configuración de los sistemas digitales en conexión con los cuales emerge para el usuario un mundo. Es necesario seguir y expandir la huella de Pariser haciendo explícitos, criticando y rediseñando el trabajo de los algoritmos más allá del terreno del acceso a la información. Su omnipresencia no sólo ha reconfigurado la imagen del bibliotecario o del gatekeeper, se ha derramado también en los espacios de conversación (Johnson, 2010), la cartografía, los juegos, el cuidado de la salud, las relaciones íntimas y una larguísima lista de 
etcéteras ${ }^{9}$. Incluso, ha impactado profundamente en las condiciones de posibilidad del aparecer del mundo.

Sin embargo, resta aún una vasta tarea: trabajar en la construcción de una relación más activa con ellos. Es necesario migrar hacia un terreno intermedio desde donde sea posible una conversación de constante redefinición de los horizontes y modalidades de mutua influencia, así como promover una relación dialógica entre los usuarios y los algoritmos.

\subsection{En diálogo con los algoritmos}

Todos tenemos una epistemología; y quien crea que no, tiene una muy mala (Bateson, 1976). De la misma forma es posible indicar hoy que todos tienen una configuración de sus extensiones digitales; y quien crea que no, tiene una muy mala. De manera que dejar por defecto el estado de las plataformas y aplicaciones sin una revisión y reflexión previa significa limitar o interrumpir la posibilidad de intervenir el modo en que se realiza esa conjugación.

\subsubsection{Código, política y retórica}

Reconocer la arquitectura subyacente delas plataformas volviendo explícita la acción del código y escrutar la capa simbólica de la que se revisten es un paso fundante hacia la construcción de una lectura crítica y propositiva. Para Lash (2005): “El código es la 'operacionalidad',la 'funcionalidad'. 'El código te permite hacer cosas'. Si el contenido es la parte frontal, el código es lo que sucede en la parte trasera (...) Las 'tuercas y tornillos' detrás de lo que hay en la pantalla” (p. 337).

Según Lessig (2001), la operacionalidad del código constituye la política de la web, entendiendo por estala configuración determinante delas posibilidades operativas de funcionamiento de un dispositivo digital. Por ejemplo, que resulte posible valorar las publicaciones en Facebook a partir de las seis reacciones disponibles (y que no sea posible hacerlo en gradientes de cero a diez, por mencionar una alternativa), es parte de su política, de su arquitectura. Que aquella valoración positiva sellame megusta en lugar de, por ejemplo, estoy de acuerdo o eres genial! forma parte dela retórica dela plataforma, ese mix de capa simbólica y programación, que Bogost (2007) bautizó como retórica procedural.

\subsubsection{Si usar es comunicar, configurar es metacomunicar}

La posibilidad de examinar ambas dimensiones habilita repensar la relación con estos dispositivos. Relación que debiera manifestarse con un carácter experimental,noestancadosino másbien abiertoala redefinición constante. AldecirdeSennet(2012), "elexperimentoinvitaala conversación dialógica,ladiscusión definalabierto con otrosacerca dehipótesis, procedimientosyresultados”(p.167).

9 No es el objetivo de este trabajo desagregar todos los ámbitos en los cuales la algoritmia ha impactado en un sentido fenomenológico. Para este tema, nos remitimos a Manovich (2013). 
Es preciso que este diálogo sea enriquecido más allá del dualismo conspirativo de programar o ser programado, sin alimentar la ilusoria posibilidad de arrancar de cuajo la indeterminación y la pasividad en la relación con la técnica (Rushkoff, 2010). Antes, más bien, asumiendo la responsabilidad de los usuarios en la administración de esas pasividades, reconociendo su participación en un encuentro comunicativo con los dispositivos, donde emerge un experienciar el mundo (Sloterdijk, 2012).

Como todo proceso comunicativo, este también ofrece la posibilidad de apertura y redefinición constante. No se trata solo de usar (comunicar), sino también, y fundamentalmente, de reflexionar acerca del tipo de relación que se está entablando (Ciuffoli \&López, 2012). Es decir, se trata de metacomunicarse (Watzlawick, Helmick Beavin \& Jackson, 1983) o comunicarse sobrela relación que se construye con los dispositivos digitales.

Sin duda, usuarios y dispositivos deben multiplicar y enriquecer sus habilidades metacomunicativas. Estas habilidades encuentran hoy en la programación programada, que es la configuración y la modificación más radical que implica la intervención directa sobre el código, dos de las fronteras que configuran los espacios de redefinición mutua. Hay mucho por delante para mejorar este diálogo.

\section{EXPERIMENTO EMPÍRICO}

En esta parte del trabajo, detenida básicamente en la experimentación, se presentan los resultados de un estudio empírico realizado -como hemos adelantado- durante la primera mitad del año 2015. Los objetivos fueron: en primer lugar, poner a prueba la afirmación de que estamos atrapados en burbujas de filtro, para lo cual se acotó la pesquisa al buscador Google, al tiempo que se buscó reflexionar sobre la presentación del buscador alternativo DuckDuckGo como un buscador estándar; en segundo lugar, se examinó el grado de conocimiento que tienen los usuarios comprendidos en la muestra acerca del funcionamiento de las cookies, las opciones de configuración y de búsqueda avanzada ofrecidas por Google, y en qué medida las intervienen.

\subsection{Detalles del experimento}

La muestra está compuesta por 49 participantes-usuarios ${ }^{\mathbf{1 0}}$. Todos ellos estudiantes de la Licenciatura en Comunicación Social de la Universidad Nacional de Rosario (UNR), que al momento del experimento se encontraban cursando el Seminario de Integración y Producción del quinto año de la carre-

10 Vale aclarar, sin embargo, que si bien el número original de participantes del experimento era de 60 usuarios, 11 de ellos fueron desestimados en el registro de las url de sus búsquedas debido a que no activaron el ítem de región en el buscador DuckDuckGo (lo cual distorsionaba la muestra), aunque fueron considerados en la segunda parte del experimento. 
$\mathrm{ra}^{11}$. Por su parte, el período en que se realizó la actividad de recolección del corpus de datos en los cuales se basa el experimento se extendió desde el 15 al 28 de mayo de $2015^{12}$. Es decir, toda la información analizada fue obtenida entre esas fechas, sin excepción.

La metodología de recolección delos datos fuela siguiente. En primerlugar, cada participante debió realizar una búsqueda usando el sintagma "elecciones 2015", en Google y en DuckDuckGo (activando en este último la opción "Región: Argentina"). La decisión de utilizar estos términos en las búsquedas estuvo motivada por la proximidad de las elecciones presidenciales de ese año. Constituyó un requisito indispensable que el término de búsqueda fuera exactamente el mismo y redactado de forma idéntica en cada buscador por cada uno de los participantes. Se sugirió realizar el ejercicio desde el ordenador que utilizaban habitualmente dado que desde allí se supone generaron una mayor cantidad de trazas en la web a partir de las cuales los buscadores puedan brindarle información personalizada.

Asimismo, cada participante debió atender a los 10 primeros resultados en ambos buscadores (exceptuando los anuncios, la información ofrecida a la derecha de los resultados y las sugerencias que suelen estar intercaladas al estilo "en las noticias"). Finalmente, luego de un detenido examen de los recursos web ofrecidos como resultado en cada buscador, cada participante debió completar un formulario ${ }^{13}$ donde se registraron las url de cada recurso, detallando a qué buscador pertenecían. También debieron responder un cuestionario.

\subsection{Operacionalizando un concepto}

Según Moretti (2014), la operacionalización es el proceso por el cual los conceptos se transforman en una serie de operaciones que habilitan la mensura de un conjunto de objetos. Operacionalizar significa "construir un puente entre los conceptos y la medición, y entre esta y el mundo" (2014, p. 166). En este caso, dos fueron los conceptos operacionalizados: burbuja de filtro y buscador genérico. En el marco de este experimento se debe entender por burbuja de filtro la operación de aislamiento informativa padecida por los usuarios de un buscador web, producto de los algoritmos de búsqueda y filtrado de la información. En cuanto al concepto de buscador genérico, debe entendérselo en el marco de este experimento como el polo opuesto al concepto de burbuja de filtro. Es decir, un buscador genérico sería aquel que da a sus usuarios ante búsquedas idénticas, idénticos resultados.

11 Se admite que el público componente del experimento puede presentar algún grado de homogeneidad al contener características comunes debilitando de este modo la validez externa del mismo. No obstante, se destaca su importancia en cuanto permite indagar con admisible consistencia en la dirección propuesta.

12 La totalidad de los datos sin procesar se encuentran disponibles en una planilla de cálculo de Google Drive en la siguiente dirección: https://goo.gl/MVVLDw

13 El formulario está disponible en: https://goo.gl/VgexsV 
Figura 1: Usuario burbujeado.

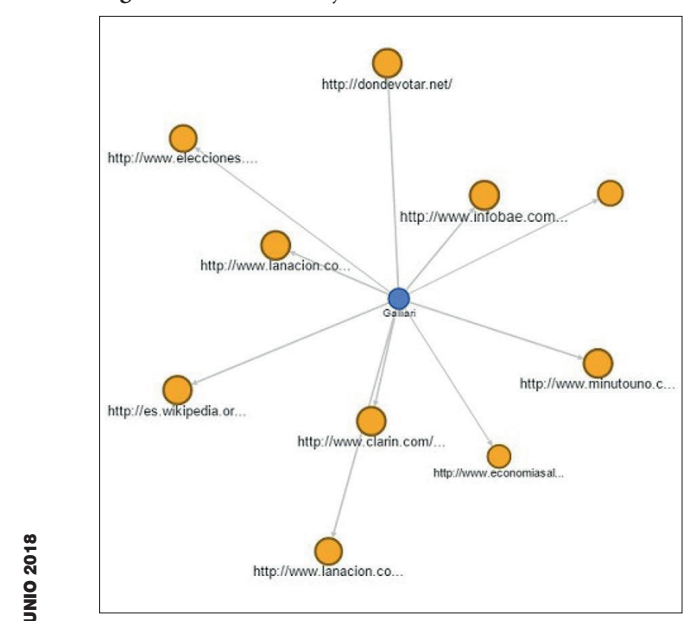

Fuente: elaboración propia.
Figura 2: Buscador genérico.

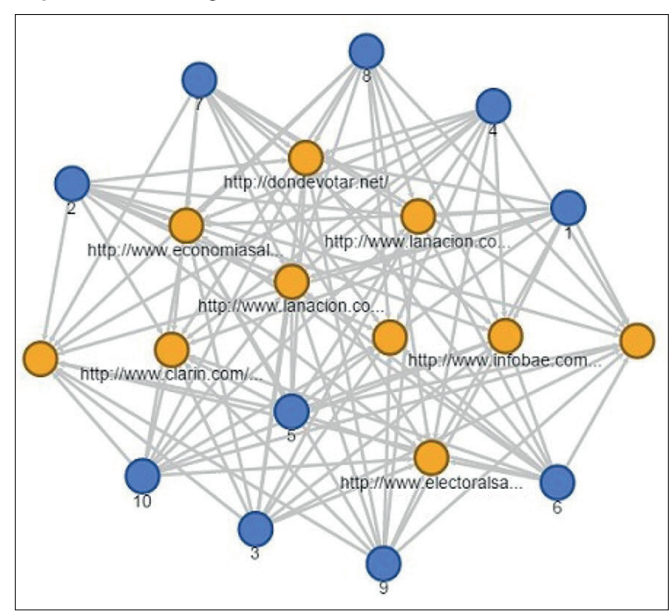

Fuente: elaboración propia.

Para esto imaginamos cómo quedarían expresadas topológicamente ambas ideas al representarlas en un grafo de red, donde:

- Cada usuario participante de la muestra sea un nodo (color azul).

- Cada uno de los resultados obtenidos en cada una de las búsquedas sea también un nodo (color amarillo).

- Cada nodo "usuario" aparezca conectado en un gráfico de red a sus nodos "resultados".

De esta manera, en el caso de que algún usuario estuviera encerrado en una burbuja de filtro por parte de alguno de los buscadores la red dela que participa, mostraría una topología del tipo estrella. Es decir, al centro de la red se ubicaría el nodo usuario y en conexión con el mismo, y sólo con ese, los diez recursos web que obtuvo como resultado de su búsqueda.

Si alguno de los usuarios que participaron de la actividad quedara representado en un grafo de topología idéntica al que muestra la Figura 1, diremos que ese usuario fue burbujeado por el buscador. En el polo opuesto a la idea de burbuja se encuentra la de un buscador genérico. Vista como un gráfico de red la comprobación de dicha situación implicaría una topología en la que solo diez recursos, y tan solo esos diez, estarían conectados con todos los participantes de la muestra.

En una situación como la ilustrada en la Figura 2 el grado de aislamiento informativo es nulo, no existe allí recurso web alguno que haya sido exclusivo de una sola búsqueda. Dicha topología indica que los mismos recursos estuvieron presentes en todas las búsquedas. 
Figura 3: Relación usuarios-recursos en Google.

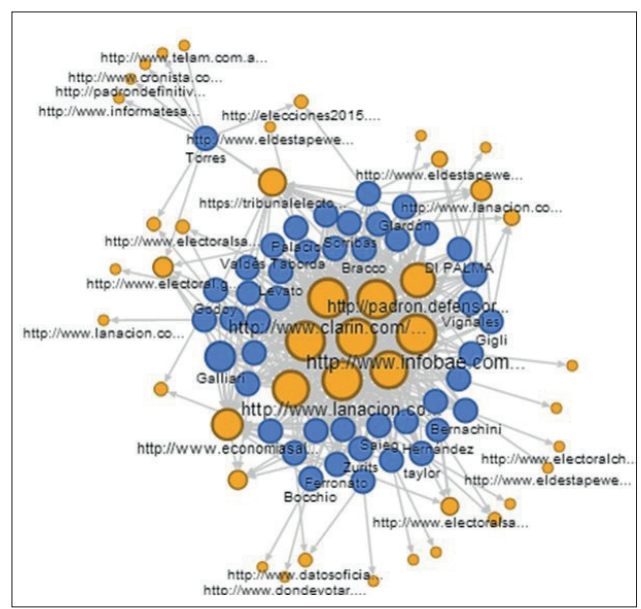

Fuente: elaboración propia.
Figura 4: Relación usuarios-recursos en DuckDuckGo.

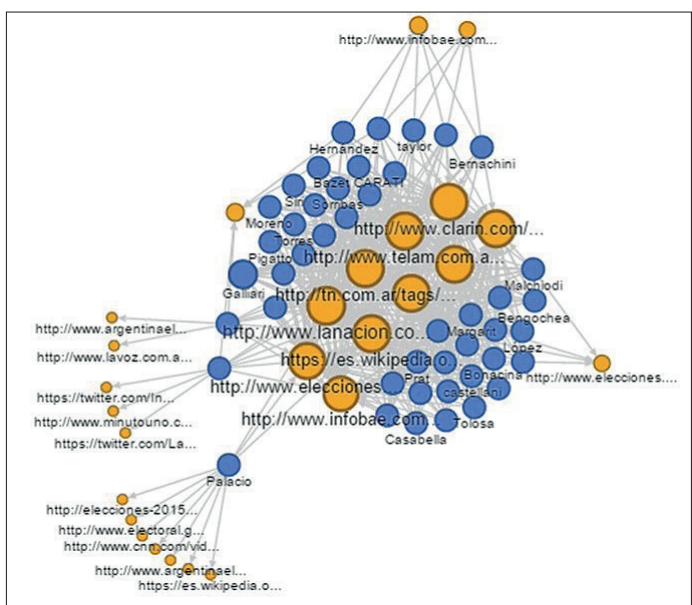

Fuente: elaboración propia.

Figura 5: Categorización de recursos según frecuencia.

\begin{tabular}{|l|l|}
\hline $\begin{array}{l}\text { Url HUB: Frecuencia de } \\
\text { aparición por encima del } \\
75 \% .\end{array}$ & $\begin{array}{l}\text { Url SATÉLITE: Su } \\
\text { frecuencia de aparición } \\
\text { oscila entre el 75\% y el } \\
25 \%\end{array}$ \\
\hline \begin{tabular}{l|l|} 
Url ISLA: Su frecuencia de \\
aparición es menor al \\
25\% pero mayor que 1
\end{tabular} & $\begin{array}{l}\text { Url BURBUJA: Frecuencia } \\
\text { de aparición igual a 1 }\end{array}$ \\
\hline
\end{tabular}

Fuente: elaboración propia.

La operacionalización de ambos conceptos fue reforzada a partir dos acciones simultáneas.

En primer lugar, se realizó una serie de gráficos de barra que permitieron visualizar las frecuencias de los diferentes recursos. Finalmente, se focalizó en el tipo y número de fuentes utilizadas por cada buscador.

\subsubsection{Distribución de las Url según el buscador}

Los gráficos de barra de las Figuras 5 y 6 fueron realizados atendiendo a la frecuencia de aparición de cada recurso (url) en cada uno de los buscadores. En estas visualizaciones se destacan el número de url que componen el total de las búsquedas y la cantidad de enlaces exclusivos de una sola búsqueda. Esto es relevante en tanto que señala el grado de aislamiento de los usuarios: 
Figura 6: Distribución según frecuencia de las Url en Google.

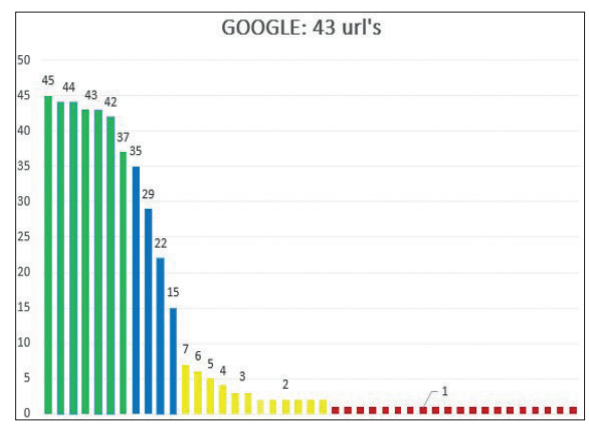

Fuente: elaboración propia.
Figura 7: Distribución según frecuencia de las Url en DuckDuckGo.

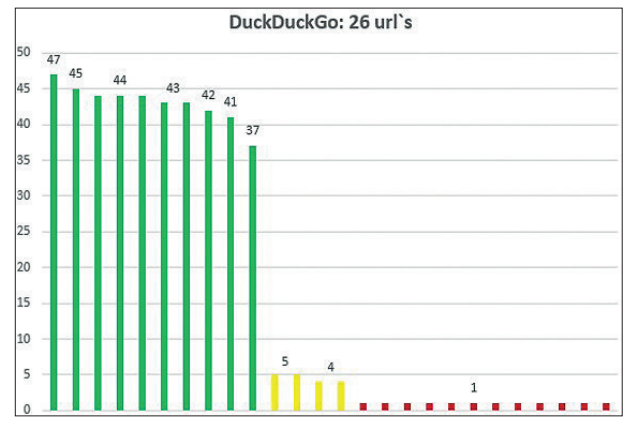

Fuente: elaboración propia.

tomando en cuenta los extremos posibles, la cantidad de enlaces exclusivos de una sola búsqueda (frecuencia $=1$ ) podría oscilar entre $490(49$ participantes x 10 recursos cada uno; y siempre de que el total de los usuarios esté completamente burbujeado), y cero, en el caso de no presentar aislamiento (véase Figura 8).

A fin de enriquecer la descripción de la distribución de los recursos, se crearon cuatro categorías en función de la frecuencia con la que aparecen en los resultados.

Asimismo, en lo referido a los usuarios "burbujeados" y el grado de aislamiento, se destaca el hecho de que ante la pregunta del cuestionario en la que tenían que decidir cuál de los buscadores les ofreció mejores resultados, los participantes burbujeados refirieron al buscador en el que no padecieron aislamiento. Cabe señalar que los usuarios burbujeados no son los mismos en ambos buscadores. Es decir, quien encontró algún tipo de aislamiento informativo en su búsqueda con Google no padeció lo mismo en DuckDuckGo, y viceversa.

Figura 8: Tabla comparativa de distribución según frecuencia.

\begin{tabular}{|l|cc|}
\hline Tipo de Url & GOOGLE & DUCKDUCKGO \\
\hline HUB & 7 & 10 \\
SATÉLITE & 4 & - \\
ISLA & 12 & 4 \\
BURBUJA & 20 & 12 \\
\hline
\end{tabular}

Fuente: elaboración propia.
Figura 9: Tabla comparativa de usuarios burbujeados y grado de aislamiento.

GOOGLE DUCKDUCKGO

\begin{tabular}{|lcc|}
\hline $\begin{array}{l}\text { Usuarios } \\
\text { burbujeados }\end{array}$ & 13 & 3 \\
$\begin{array}{l}\text { Usuario más } \\
\text { aislado }\end{array}$ & $\begin{array}{r}\text { 5 Url's } \\
\text { burbuja }\end{array}$ & $\begin{array}{l}\text { 7 Url's } \\
\text { burbuja }\end{array}$ \\
\hline
\end{tabular}

Fuente: elaboración propia. 
Figura 10: Variedad y frecuencia de sitios raíz en Google.

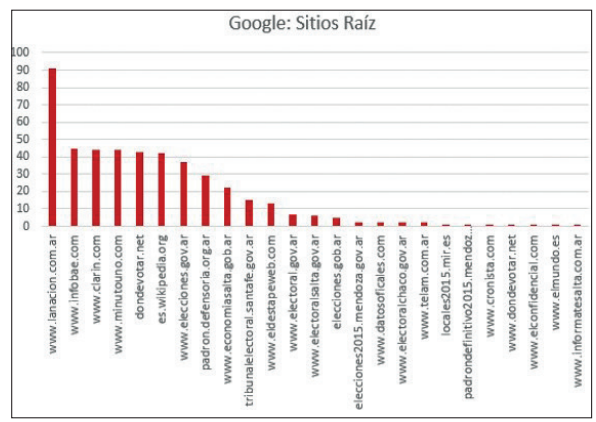

Fuente: elaboración propia.
Figura 11: Variedad y frecuencia de sitios raíz en DuckDuckGo.

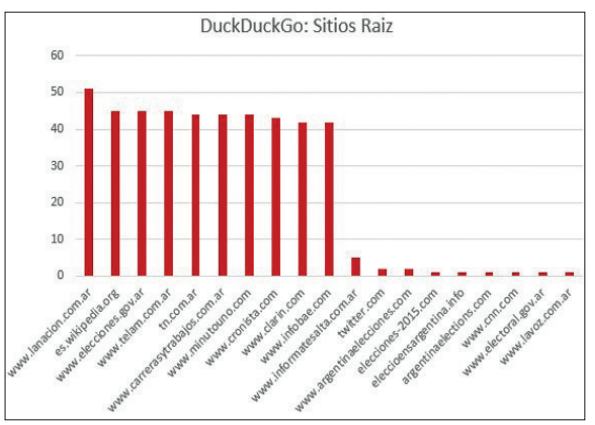

Fuente: elaboración propia.

\subsubsection{Sitios raíz y fuentes compartidas}

Los dos gráficos que siguen reflejan la variedad y recurrencia de los sitios raíz manejados por ambos buscadores para elaborar sus resultados. Seentiende por sitio raíz lo siguiente: si la dirección absoluta de un recurso es: http://www. lanacion.com.ar/elecciones-2015-t50499, entonces decimos que su fuente, su sitio raíz es: http://www.lanacion.com.ar. Se hacen visibles también cuántas y cuáles de estas fuentes tienen en común ambos buscadores.

Por su parte, y a partir del experimento realizado, podemos determinar que los buscadores no han utilizado exactamente las mismas fuentes y no las han ponderado (alas fuentes compartidas) de igual manera. Aun tomando en cuenta el sitio de noticias www.lanacion.com.ar al que ambos buscadores sitúan en el primer puesto del ranking de frecuencias, vemos que lo hacen de maneras muy disímiles: se encuentra muy destacado en el caso de Google y apenas destacado entre las diez fuentes principales en DuckDuckGo. En tal sentido, vale precisar que los sitios raíz compartidos por ambos buscadores fueron 10 :

Figura 12: Tabla sitios raíz compartidos por ambos buscadores.

\begin{tabular}{|c|l|c|l|}
\hline 1 & http://www.lanacion.com.ar & 6 & http://www.elecciones.gov.ar \\
\hline 2 & http://es.wikipedia.org & 7 & http://www.telam.com.ar \\
\hline 3 & http://www.infobae.com & 8 & http://www.cronista.com \\
\hline 4 & http://www.minutouno.com & 9 & http://www.electoral.gov.ar \\
\hline $\mathbf{5}$ & http://www.clarin.com & 10 & http://www.informatesalta.com.ar \\
\hline
\end{tabular}

Fuente: elaboración propia. 


\subsection{Opciones de búsqueda, cookies y configuración}

\begin{tabular}{|c|c|}
\hline $\begin{array}{l}\text { ¿Cuál de los dos buscadores te ofreció } \\
\text { resultados más satisfactorios? }\end{array}$ & $\begin{array}{c}\text { ¿Usás las opciones de búsqueda } \\
\text { avanzada? }\end{array}$ \\
\hline Figura 13.1 & Figura 13.2 \\
\hline DuckDuckGo: 31 - Google: 25 & $\begin{array}{l}\text { Sí, las utilizo: } 28 \text { - Sé que existen } \\
\text { pero no las uso: } 23 \text { - No sabía que } \\
\text { existían: } 5\end{array}$ \\
\hline $\begin{array}{l}\text { ¿Conocés las opciones de } \\
\text { configuración del buscador? }\end{array}$ & ¿Sabés cómo funcionan las Cookies? \\
\hline Figura 13.3 & Figura 13.4 \\
\hline $\begin{array}{l}\text { Sé que existen pero no las uso: } \\
37 \text { - Sí, las utilizo: } 14 \text { - No sabía que } \\
\text { existían: } 5\end{array}$ & $\begin{array}{l}\text { Sí, sé cómo funcionan pero nunca } \\
\text { las modifiqué: } 33 \text { - No tengo idea } \\
\text { de cómo funcionan: } 13 \text { - ¿Dijiste } \\
\text { Cookies?: } 1\end{array}$ \\
\hline
\end{tabular}

Fuente: elaboración propia.

\section{CONSIDERACIONES FINALES}

Los resultados del experimento no ofrecen un escenario empírico contundente que permita afirmar, en un sentido estricto, que el buscador de Google aisló informativamente a los participantes de la muestra. Asílo indica el corpus total de 43 url utilizadas para confeccionar los resultados de las búsquedas, de las cuales siete fueron del tipo hub ${ }^{14}$; es decir, aquellas que lejos de aislar a los usuarios contribuyen a configurar un mapa informativo común (Véase Figura $3)$. En cuanto a las url satélite, las cuales operan en un grado intermedio de concentración, la cantidad fue de cuatro, mientras que las url tipo isla alcanzaron un total de doce. Si bien no llegan a representar un cabal aislamiento, estas

14 En cuanto a las características de la tipología propuesta para las url de acuerdo a su frecuencia de aparición en los resultados de las búsquedas: véase la Figura 5. 
últimas operaron una importante dispersión en el mapa informativo (figura 3). Por último, la cantidad de url del tipo burbuja (figura 5), exclusivas de una única búsqueda y que sí generan un aislamiento contundente, trepó a veinte y se repartieron entre trece usuarios. A saber: un usuario con cinco url burbuja, tres usuarios con dos y nueve usuarios con una.

En cuanto al buscador alternativo DuckDuckGo, su corpus total de url fue de 26, respondiendo a la siguiente distribución según la tipología planteada: 10 url del tipo hub, cuatro del tipo isla y 12 burbuja de las cuales siete recayeron en la búsqueda de un solo usuario (convirtiéndose en el más aislado de toda la muestra: véase Figura 9), repartiéndose las restantes entre dos usuarios con tres y dos url burbuja en cada. No obstante, presentar la mayor reunión de url burbuja en una única búsqueda, DuckDuckGo estuvo muy cerca de constituirse en un buscador genérico (ante búsquedas idénticas ofrece idénticos resultados). Tal como lo manifiesta el gráfico de red de la Figura 4 configuró para los usuarios un territorio informativo común muy concentrado, acompañado de una baja presencia de url de frecuencia intermedia (isla y satélite) y alta concentración de las url burbuja en un único participante (véase Figura 5).

En relación a los resultados y el tratamiento de los sitios raíz, se observa una diferencia en el volumen del corpus utilizado en cada caso (25 Google y 19 DuckDuckGo), y una coincidencia en cuanto ambos buscadores ponderaron en primer lugar la misma fuente: www.lanacion.com.ar. Sin embargo, DuckDuckGo despliega una distribución más equilibrada donde las primeras diez fuentes se reparten, sin demasiada diferencia entre sí, más del 90\% del total de las impresiones (véase Figura 11). Elbuscador de Google, por su parte, presenta una gran concentración en el sitio raíz: www.lanacion.com.ar, el cual dobla en frecuencia (91 sobre 45) al que se sitúa en segunda posición (www.infobae. com). En cuanto a los sitios raíz compartidos, la cantidad total asciende a diez, de los cuales tan solo tres se encuentran entre los de mayor frecuencia: www. lanacion.com.ar, es.wikipedia.org y www.elecciones.gov.ar.

En torno a estos fenómenos, permanece latente la necesidad de preguntarnos en qué medida la personalización implica aislamiento en un sentido rotundo, o si puede considerarse algún umbral por debajo del cual la divergencia informativa sea comprendida positivamente. Para esto es menester profundizar en el tipo de categorización planteada a partir de este experimento y sus implicancias en la constitución de territorios informativos compartidos.

En cuanto a la mejora de la relación dialógica con los dispositivos, los resultados del experimento muestran una clara tendencia: la gran mayoría de los participantes acusa tener conocimiento de la existencia y/o el modo de operar de las cookies detrás de sus acciones, así como las opciones avanzadas de su buscador (indispensables para intervenir el rumbo por defecto de una búsqueda). Sin embargo, fueron muy pocos los que manifestaron estar dispuestos a hacer efectiva esa modificación. Por el contrario, la actitud que prima es la 
de la no intervención. De manera que en el marco de esta experiencia no ha sido el desconocimiento sino la indiferencia la que alejó a los participantes de la posibilidad de metacomunicarse con sus dispositivos.

Cuestiones, todas, que plantean la necesidad de retomar el fenómeno analizado y seguir desarrollando estudios capaces de ampliar la reflexión a partir de experiencias de mayor complejidad y alcance empírico.

\section{REFERENCIAS}

Barabasi, L. (2003). Linked. New York: Perseus Books.

Bateson, G. (1976). Pasos hacia una ecología de la mente. Buenos Aires: Carlos Lohlé.

Berners Lee, T. \& Fischetti, M. (2000). Tejiendo la red: el inventor de la world wide web nos descubre su origen. Madrid: Siglo XXI.

Bogost, I. (2007). Persuasive Games. The expressivepower ofvideo games. Massachusetts: MIT Press.

Borges, J. L. (1994). El pudor de la historia. En Otras inquisiciones. Buenos Aires: Emecé.

Ciuffoli, C. \& López, G. (2012). Facebook es el mensaje. Buenos Aires: La Crujía.

Cobo, A., Gómez, P., Pérez, D. \& Rocha, R. (2005). PHP y MySQL. Tecnologías para el desarrollo deaplicaciones web. Madrid:Editorial DíazdeSantosS. A. DíazdeSantos.

Deleuze, G. (2008). Kant y el tiempo. Buenos Aires: Cactus.

García Moggia, M. (2014). Breve historia de la ventana. Laboratorio. Recuperado: 02/02/2018. En línea: http://revistalaboratorio.udp.cl/num9_2014_art7_garcia/

Igarza, R. (2008). Nuevos medios. Estrategias de convergencia. Buenos Aires: La Crujía.

Johnson, S. (2010) ¿De dónde provienen las buenas ideas? Recuperado: 10/02/18. En línea: http://www.ted.com/talks/steven_johnson_where_good_ideas_come_ from?language $=\mathrm{es}$

Lash, S. (2005). Crítica de la información. Madrid: Amorrortu. Lash, S. (2005). Capitalismo y metafísica. En Arfuch, L. (comp.). Pensar este tiempo. Espacios, afectos, pertenencias (pp. 47-74). Buenos Aires: Paidós.

Lessig, L. (2009). El código 2.0. Madrid: Traficantes de Sueños.

Lewkowicz, I. (2004). Pensar sin Estado. La subjetividad en la era de la fluidez. Buenos Aires: Paidós.

Manovich, L. (2013). El software toma el mando. Cataluña: Editorial UOC.

McLuhan, E. \& McLuhan, M. (1990). Leyes de los medios. La nueva ciencia. México, D.F.: Alianza. 
Moretti, F. (2014). Operacionalizar. New Left Review, 84, 115-132.

Pariser, E. (2011). Cuidado con las burbujas de filtro. Conferencia TED. Recuperado: 20/11/2017 En línea: http://www.ted.com/talks/eli_pariser_beware_online_ filter_bubbles?language $=\mathrm{es}$

Pariser, E. (2017). El filtro burbuja. Cómo la red decide lo que leemos y lo que pensamos. Barcelona: Taurus.

Pickhardt, R. (11 de mayo de 2011). What are the 57 signals google uses to filter search results? Archive Today. Recuperado:24/02/2018. En línea: https://archive.is/IgkoJ

Resnick, M. (2001). Tortugas, Termitas y Atascos de Tráfico: Exploraciones sobre micromundos masivamente paralelos. Barcelona: Gedisa.

Rushkoff, D. (2010). Program or Be Programmed: Ten Commands for a Digital Age. New York: OR Books

Schwartz, J. (4 de septiembre de 2001). Giving Web a Memory Cost Its Users Privacy. The New York Times. Recuperado: 01/04/2017. En línea: https://www.nytimes. com/2001/09/04/business/giving-web-a-memory-cost-its-users-privacy.html

Sennet, R. (2012). Juntos. Rituales, placeres y política de cooperación. Barcelona: Anagrama.

Sloterdijk, P. (2012). Has de cambiar tu vida. Valencia: Pre-Textos.

Watts, D. (2006). Seis grados de separación. Buenos Aires: Paidós.

Watzlawick, P., Helmick Beavin, J. \& Jackson, D. (1983). Teoría de la comunicación humana. Barcelona: Herder.

\section{IDENTIFICACIÓN DEL AUTOR}

Aníbal Guillermo Rossi es Licenciado en Comunicación Social por la Universidad Nacional de Rosario (UNR), Argentina. Actualmente, se encuentra cursando la Maestría en Comunicación Digital Interactiva, UNR. Es docente de la cátedra Seminario de Integración y Producción de la Licenciatura en Comunicación Social (UNR), de la cátedra Tecnologías Digitales de la Comunicación y la Información en el Postítulo en Periodismo y Comunicación(UNR), y en asignaturas diversas de Ingeniería en Sistemas Informáticos y la Facultad de Comunicación de la Universidad Abierta Interamericana (UAI), Argentina. Coordina el laboratorio interdisciplinar de investigación y producción experimental en narrativas digitales "Narrativas Lab" (http://www.narrativaslab.com). Ha dictado cursos y conferencias sobre alfabetización procedural, entre las que destaca "Viejos narcisos y nuevos reflejos. Lo que la educación puede aprender del software” (TEDx Rosario 2013).

\section{REGISTRO BIBLIOGRÁFICO}

Rossi, A. (enero-junio, 2018). ¿Burbujas de filtro? Hacia una fenomenología algorítmica. InMediaciones de la Comunicación, 13(1), 263-281 